\title{
Survival and Stationary Distribution in a Stochastic SIS Model
}

\author{
Yanli Zhou, ${ }^{1,2}$ Weiguo Zhang, ${ }^{1}$ and Sanling Yuan' \\ ${ }^{1}$ College of Science, University of Shanghai for Science and Technology, Shanghai 200093, China \\ ${ }^{2}$ Shanghai Medical Instrumentation College, Shanghai 200093, China \\ Correspondence should be addressed to Sanling Yuan; sanling@usst.edu.cn
}

Received 10 October 2013; Accepted 12 November 2013

Academic Editor: Zhen Jin

Copyright (C) 2013 Yanli Zhou et al. This is an open access article distributed under the Creative Commons Attribution License, which permits unrestricted use, distribution, and reproduction in any medium, provided the original work is properly cited.

\begin{abstract}
The dynamics of a stochastic SIS epidemic model is investigated. First, we show that the system admits a unique positive global solution starting from the positive initial value. Then, the long-term asymptotic behavior of the model is studied: when $R_{0} \leq 1$, we show how the solution spirals around the disease-free equilibrium of deterministic system under some conditions; when $R_{0}>1$, we show that the stochastic model has a stationary distribution under certain parametric restrictions. In particular, we show that random effects may lead the disease to extinction in scenarios where the deterministic model predicts persistence. Finally, numerical simulations are carried out to illustrate the theoretical results.
\end{abstract}

\section{Introduction}

Mathematical epidemiology describing the population dynamics of infectious diseases has been made a significant progress in better understanding of disease transmissions and behavior of epidemics. Many epidemic models have been described by ordinary differential equations [1-11]. These important and useful deterministic investigations offer a great insight into the effects of infectious disease, but in the real world, epidemic dynamics is inevitably affected by the environmental noise, which is an important component in the epidemic systems. As a matter of fact, the epidemic models are often subject to environmental noise; that is, due to environmental fluctuations, parameters involved in epidemic models are not absolute constants, and they may fluctuate around some average values. So, inclusion of random perturbations in such models makes them more realistic in comparison to their deterministic counterparts. In recent years, epidemic models under environmental noise described by stochastic different equations have been studied by many researchers. They introduce stochastic noises into deterministic models to reveal the effect of environmental variability on the epidemic dynamics in mathematical ecology [12-20]. For example, Chen and $\mathrm{Li}$ [12] discussed the stability of the endemic equilibrium of a stochastic SIR model. Tornatore et al. [13] studied the stability of the disease-free equilibrium of a stochastic SIR model with and without distributed time delay. Ji et al. [14] discussed a multigroup SIR model with perturbation, where they showed that if the basic reproduction number $R_{0} \leq 1$, then the solution of the model oscillates around the disease-free steady state, whereas, if $R_{0}>1$, there is a stationary distribution. However, this field is still in its infancy.

The classical epidemic models proposed by Kermack and McKendrick in 1927 [21] have been extensively investigated in the literature. The deterministic SIS model takes the following form:

$$
\begin{aligned}
& \dot{S(t)}=\Lambda-\lambda S(t) I(t)-\mu S+\gamma I(t), \\
& \dot{I(t)}=\lambda S(t) I(t)-(\gamma+\mu+\epsilon) I(t),
\end{aligned}
$$

where $S(t)$ represents the number of susceptible individuals and $I(t)$ denotes the number of infected individuals at time $t$. The influx of individuals into the susceptible is given by a constant $\Lambda . \mu$ is the natural death rate; $\epsilon$ represents the death rate due to disease; $\lambda$ and $\gamma$ represent the disease transmission coefficient and the rate of recovery from infection, respectively. Model (1) always has a disease-free equilibrium $E^{0}=$ $\left(S^{0}, I^{0}\right)=(\Lambda / \mu, 0)$ and it is globally asymptotical stable if the basic reproduction number $R_{0}=\Lambda \lambda / \mu(\mu+\epsilon+\gamma) \leq 1$, which means the disease will disappear and the entire population will become susceptible. When $R_{0}>1, E^{0}$ becomes unstable 
and a globally asymptotical stable endemic equilibrium $E^{*}=$ $\left(S^{*}, I^{*}\right)=((\gamma+\mu+\epsilon) / \lambda,(\Lambda \lambda-\mu(\gamma+\mu+\epsilon)) / \lambda(\mu+\epsilon))$ appears, which implies that the disease will always prevail and persist in the population.

As stated above, due to the existence of environmental noise, the parameters of model (1) are not absolute constants, and they exhibit continuous oscillation around some average values but do not attain fixed values with the advancement of time. Considering the biological significance and the effects of stochastic environmental noise perturbations, there are different possible approaches to include random effects in the model. The following four approaches are usually adopted in the literature (see [22]). The first one is through time Markov chain model to consider environment noise [23]. The second is with parameters perturbation [24]. The third one is the environmental noise that is proportional to the variables [25], and the last one is to robust the positive equilibria of deterministic models. In this paper, we will consider a stochastic counterpart of model (1) in a combination of the second and third approaches. That is, the stochastic perturbation is assumed to be of a white noise type which is directly proportional to $S(t), I(t)$, influenced respectively on $\dot{S(t)}$ and $I \dot{(t)}$ in model (1); meantime, the disease transmission coefficient $\lambda$ in model (1) is replaced by $\lambda+\sigma_{3} \dot{B}_{3}(t)$. Our model takes the following form:

$$
\begin{aligned}
\mathrm{d} S(t)= & (\Lambda-\lambda S(t) I(t)-\mu S(t)+\gamma I(t)) \mathrm{d} t \\
& +\sigma_{1} S(t) \mathrm{d} B_{1}(t)-\sigma_{3} S(t) I(t) \mathrm{d} B_{3}(t), \\
\mathrm{d} I(t)= & {[\lambda S(t) I(t)-(\gamma+\mu+\epsilon) I(t)] \mathrm{d} t } \\
& +\sigma_{2} I(t) \mathrm{d} B_{2}(t)+\sigma_{3} S(t) I(t) \mathrm{d} B_{3}(t),
\end{aligned}
$$

where $B_{1}(t), B_{2}(t)$, and $B_{3}(t)$ are mutually independent Brownian motions and $\sigma_{1}, \sigma_{2}$, and $\sigma_{3}$ are noise intensities.

The organization of this paper is as follows. In Section 2, we show that there is a unique positive solution of model (2) for any positive initial value. In Section 3, we show how the solution spirals around the disease-free equilibrium of deterministic system under some conditions. In Section 4, we prove that model (2) has a stationary distribution under certain parametric restrictions. In Section 5, an extinction result due to large noises is presented. Finally, numerical simulations are carried out to illustrate the main theoretical results.

\section{Existence and Uniqueness of the Positive Solution}

Throughout this paper, let $\left(\Omega, \mathscr{F},\left\{\mathscr{F}_{t}\right\}_{t \geq 0}, P\right)$ be a complete probability space with a filtration satisfying the usual conditions (i.e., it is right continuous and $\mathscr{F}_{0}$ contains all $P$-null sets). Let $B_{i}(t)(i=1,2,3)$ denote the independent standard Brownian motions defined on this probability space. We also denote

$$
\mathbb{R}_{+}^{2}=\left\{(x, y) \in \mathbb{R}^{2}: x>0, y>0\right\} .
$$

Since the $S(t)$ and $I(t)$ in model (2) are, respectively, the sizes of the susceptible individuals and the infected individuals at time $t$, they should be nonnegative. Therefore, we are only interested in positive solutions. In order to get a unique global solution (i.e., no explosion in a finite time) for any given initial value, the coefficients of the stochastic differential equation are generally required to satisfy the linear growth condition and local Lipschitz condition [26]. However, the coefficients of model (2) do not satisfy the linear growth condition, though they are locally Lipschitz continuous; hence the solution of model (2) may explode at a finite time. In what follows, we shall prove that the solution of model (2) is positive and global.

Theorem 1. For any given initial condition $(S(0), I(0)) \in \mathbb{R}_{+}^{2}$, there is a unique positive solution $(S(t), I(t))$ of model (2) on $t \geq 0$, and the solution will remain in $\mathbb{R}_{+}^{2}$ with probability one, namely, $(S(t), I(t)) \in \mathbb{R}_{+}^{2}$ for all $t \geq 0$ a.s.

Proof. Since the coefficients of the equation are locally Lipschitz continuous, for any initial value $(S(0), I(0)) \in \mathbb{R}_{+}^{2}$, there is a unique local solution $(S(t), I(t))$ on $t \in\left[0, \tau_{e}\right)$, where $\tau_{e}$ is the explosion time. To show this solution is global, we need to show that $\tau_{e}=+\infty$ a.s. Define the stopping time

$$
\tau^{+}=\inf \left\{t \in\left[0, \tau_{e}\right): S(t) \leq 0, I(t) \leq 0\right\},
$$

where throughout this paper we set inf $\phi=\infty$ (as usual $\phi$ denotes the empty set). Obviously, we have $\tau^{+} \leq \tau_{e}$. So, if we can prove that $\tau^{+}=\infty$ a.s., then $\tau_{e}=\infty$ and $(S(t), I(t)) \in \mathbb{R}_{+}^{2}$ a.s. for all $t \geq 0$.

Assume that $\tau^{+}<\infty$; then there exists a $T>0$ such that $\mathbb{P}\left(\tau^{+}<T\right)>0$. Define a $C^{2}$-function $V: \mathbb{R}_{+}^{2} \rightarrow \mathbb{R}_{+}$by

$$
V(S(t), I(t))=\ln S(t) I(t) .
$$

Applying Itô's formula, we can get

$$
\begin{aligned}
\mathrm{d} V(S(t), I(t))= & \frac{1}{S(t)} \mathrm{d} S(t)-\frac{1}{2 S^{2}(t)}(\mathrm{d} S(t))^{2} \\
& +\frac{1}{I(t)} \mathrm{d} I(t)-\frac{1}{2 I^{2}(t)}(\mathrm{d} I(t))^{2} .
\end{aligned}
$$

That is,

$$
\begin{aligned}
\mathrm{d} V & (S(t), I(t)) \\
= & {\left[\frac{\Lambda}{S(t)}-(2 \mu+\epsilon+\gamma)+\frac{\gamma I(t)}{S(t)}+\lambda S(t)\right.} \\
& \left.-\lambda I(t)-\frac{\sigma_{1}^{2}+\sigma_{2}^{2}}{2}-\frac{\sigma_{3}^{2} I^{2}(t)+\sigma_{3}^{2} S^{2}(t)}{2}\right] \mathrm{d} t \\
& +\sigma_{1} \mathrm{~d} B_{1}(t)+\sigma_{2} \mathrm{~d} B_{2}(t) \\
& -\sigma_{3} I(t) \mathrm{d} B_{3}(t)+\sigma_{3} S(t) \mathrm{d} B_{3}(t) .
\end{aligned}
$$

Due to the positivity of $S(t)$ and $I(t)$, it follows from the above equality that

$$
\begin{gathered}
\mathrm{d} V(S(t), I(t)) \geq k(S(t), I(t)) \mathrm{d} t+\sigma_{1} \mathrm{~d} B_{1}(t)+\sigma_{2} \mathrm{~d} B_{2}(t) \\
-\sigma_{3} I(t) \mathrm{d} B_{3}(t)+\sigma_{3} S(t) \mathrm{d} B_{3}(t),
\end{gathered}
$$


where $k(S(t), I(t))=-(2 \mu+\epsilon+\gamma+\lambda I(t))-\left(\sigma_{1}^{2}+\sigma_{2}^{2}\right) / 2-$ $\left(\sigma_{3}^{2} I^{2}(t)+\sigma_{3}^{2} S^{2}(t)\right) / 2$.

Integrate both sides of (8) from 0 to $t$; we yield

$V(S(t), I(t)) \geq V(S(0), I(0))$

$$
\begin{aligned}
& +\int_{0}^{t} k(S(u), I(u)) \mathrm{d} u+\sigma_{1} B_{1}(t)+\sigma_{2} B_{2}(t) \\
& -\int_{0}^{t} \sigma_{3} I(u) \mathrm{d} B_{3}(u)+\int_{0}^{t} \sigma_{3} S(u) \mathrm{d} B_{3}(u) .
\end{aligned}
$$

It is easy to see that, for almost all $\omega$ in $\tau^{+}<\tau_{e}, S(t), I(t)$ are positive on $\left[0, \tau^{+}\right)$and $S\left(\tau^{+}\right) I\left(\tau^{+}\right)=0$. Accordingly,

$$
\lim _{t \rightarrow \tau^{+}}[\ln S(t)+\ln I(t)]=-\infty .
$$

Letting $t \rightarrow \tau^{+}$, it follows from (9) that

$$
\begin{aligned}
-\infty \geq V & (S(0), I(0)) \\
& +\int_{0}^{\tau^{+}} k(S(t), I(t)) \mathrm{d} u+\sigma_{1} B_{1}\left(\tau^{+}\right)+\sigma_{2} B_{2}\left(\tau^{+}\right) \\
& -\int_{0}^{\tau^{+}} \sigma_{3} I(t) \mathrm{d} B_{3}(u)+\int_{0}^{\tau^{+}} \sigma_{3} S(t) \mathrm{d} B_{3}(u)>-\infty
\end{aligned}
$$

which is a contradiction; therefore we have $\tau^{+}=\infty$ a.s. The proof is therefore completed.

\section{Asymptotic Behavior around the \\ Disease-Free Equilibrium $E^{0}$}

As mentioned above, if $R_{0} \leq 1$, then model (1) always has a globally asymptotically stable disease-free equilibrium $E^{0}$, which means the disease will die out with the advancement of time. Noting that $E^{0}$ is not an equilibrium of stochastic model (2), it is natural to ask whether the disease will go to extinction in the population. In this section we mainly use the way of estimating the oscillation around $E^{0}$ to reflect how the solution of model (2) spirals closely around $E^{0}$. We have the following theorem.

Theorem 2. Assume $R_{0} \leq 1$. Then for any solution $(S(t), I(t))$ of model (2) with initial value $(S(0), I(0)) \in \mathbb{R}_{+}^{2}$, if $\sigma_{1}^{2}<\mu$ and $\sigma_{2}^{2} / 2<\mu+\epsilon$, we have

$$
\limsup _{t \rightarrow \infty} \frac{1}{t} E \int_{0}^{t}\left[\left(S(u)-\frac{\Lambda}{\mu}\right)^{2}+I^{2}(u)\right] \mathrm{d} u \leq \frac{\sigma_{1}^{2} \Lambda^{2}}{m \mu^{2}},
$$

where $m=\min \left\{\mu-\sigma_{1}^{2}, \mu+\epsilon-\sigma_{2}^{2} / 2\right\}$.

Proof. Define a function $V: \mathbb{R}_{+}^{2} \rightarrow \mathbb{R}_{+}$by

$$
\begin{aligned}
V(t) & =V_{1}(t)+\frac{2 \mu+\epsilon}{\lambda} V_{2}(t) \\
& =\frac{(S(t)-(\Lambda / \mu)+I(t))^{2}}{2}+\frac{2 \mu+\epsilon}{\lambda} I(t) .
\end{aligned}
$$

Along the trajectories of system (2), we have

$$
\begin{aligned}
& \mathrm{d} V_{1}(t)=\left(S(t)-\frac{\Lambda}{\mu}+I(t)\right)(\mathrm{d} S(t)+\mathrm{d} I(t)) \\
&+\frac{1}{2}(\mathrm{~d} S(t)+\mathrm{d} I(t))^{2} \\
&= L V_{1}(t) \mathrm{d} t+\left(S(t)-\frac{\Lambda}{\mu}+I(t)\right) \\
& \times\left(\sigma_{1} S(t) \mathrm{d} B_{1}(t)+\sigma_{2} I(t) \mathrm{d} B_{2}(t)\right), \\
& \mathrm{d} V_{2}(t)=L V_{2}(t) \mathrm{d} t+\sigma_{2} I(t) \mathrm{d} B_{2}(t)+\sigma_{3} S(t) I(t) \mathrm{d} B_{3}(t),
\end{aligned}
$$

where

$$
\begin{aligned}
L V_{1}(t)= & \left(S(t)-\frac{\Lambda}{\mu}+I(t)\right)(\Lambda-\mu S(t)-(\mu+\epsilon) I(t)) \\
& +\frac{\sigma_{1}^{2} S^{2}(t)+\sigma_{2}^{2} I^{2}(t)}{2} \\
& L V_{2}(t)=\lambda S(t) I(t)-(\mu+\gamma+\epsilon) I(t) .
\end{aligned}
$$

From (15), we have that

$$
\begin{aligned}
L V_{1}(t) & \\
= & \left(S(t)-\frac{\Lambda}{\mu}+I(t)\right)\left[-\mu\left(S(t)-\frac{\Lambda}{\mu}\right)-(\mu+\epsilon) I(t)\right] \\
& +\frac{\sigma_{1}^{2}(S(t)-\Lambda / \mu+\Lambda / \mu)^{2}+\sigma_{2}^{2} I^{2}(t)}{2} \\
\leq & -\left(\mu-\sigma_{1}^{2}\right)\left(S(t)-\frac{\Lambda}{\mu}\right)^{2}-(2 \mu+\epsilon)\left(S(t)-\frac{\Lambda}{\mu}\right) I(t) \\
& -\left(\mu+\epsilon-\frac{\sigma_{2}^{2}}{2}\right) I^{2}(t)+\sigma_{1}^{2}\left(\frac{\Lambda}{\mu}\right)^{2},
\end{aligned}
$$

where in the last step we have used the inequality $(a+b)^{2} \leq$ $2 a^{2}+2 b^{2}$. Similarly, we can have from (16) that

$$
\begin{aligned}
L V_{2}(t) & =\lambda\left(S(t)-\frac{\Lambda}{\mu}+\frac{\Lambda}{\mu}\right) I(t)-(\mu+\gamma+\epsilon) I(t) \\
& =\lambda\left(S(t)-\frac{\Lambda}{\mu}\right) I(t)+\left[\lambda \frac{\Lambda}{\mu}-(\mu+\gamma+\epsilon)\right] I(t) .
\end{aligned}
$$

Noting that $R_{0}=\Lambda \lambda / \mu(\mu+\epsilon+\gamma) \leq 1$, it follows from the above that

$$
L V_{2}(t) \leq \lambda\left(S(t)-\frac{\Lambda}{\mu}\right) I(t) .
$$


It follows from (17), (19), and (13) that

$$
\begin{aligned}
L V(t) \leq & -\left(\mu-\sigma_{1}^{2}\right)\left(S(t)-\frac{\Lambda}{\mu}\right)^{2} \\
& -\left(\mu+\epsilon-\frac{\sigma_{2}^{2}}{2}\right) I^{2}(t)+\sigma_{1}^{2}\left(\frac{\Lambda}{\mu}\right)^{2} .
\end{aligned}
$$

Integrating from 0 to $t$ on both sides of (20) and taking expectation yield

$$
\begin{aligned}
E V(t)-V(0) \leq & -\left(\mu-\sigma_{1}^{2}\right) E \int_{0}^{t}\left(S(u)-\frac{\Lambda}{\mu}\right)^{2} \mathrm{~d} u \\
& -\left(\mu+\epsilon-\frac{\sigma_{2}^{2}}{2}\right) E \int_{0}^{t} I^{2}(u) \mathrm{d} u+\sigma_{1}^{2}\left(\frac{\Lambda}{\mu}\right)^{2} t .
\end{aligned}
$$

Hence, we have that

$$
\begin{aligned}
\limsup _{t \rightarrow \infty} \frac{1}{t} E \int_{0}^{t} & {\left[\left(\mu-\sigma_{1}^{2}\right)\left(S(u)-\frac{\Lambda}{\mu}\right)^{2}\right.} \\
+ & \left.\left(\mu+\epsilon-\frac{\sigma_{2}^{2}}{2}\right) I^{2}(u)\right] \mathrm{d} u \leq \sigma_{1}^{2}\left(\frac{\Lambda}{\mu}\right)^{2} .
\end{aligned}
$$

Let $m=\min \left\{\mu-\sigma_{1}^{2}, \mu+\epsilon-\sigma_{2}^{2} / 2\right\}$; it follows from (22) that

$$
\limsup _{t \rightarrow \infty} \frac{1}{t} E \int_{0}^{t}\left[\left(S(u)-\frac{\Lambda}{\mu}\right)^{2}+I^{2}(u)\right] \mathrm{d} u \leq \frac{\sigma_{1}^{2} \Lambda^{2}}{m \mu^{2}} .
$$

The proof of Theorem 2 is thus completed.

Notice that when $\sigma_{1}=0, E^{0}$ is also the disease-free equilibrium of system (2), and (20) is reduced to the following form:

$$
L V(t) \leq-\mu\left(S(t)-\frac{\Lambda}{\mu}\right)^{2}-\left(\mu+\epsilon-\frac{\sigma_{2}^{2}}{2}\right) I^{2}(t),
$$

which means that $L V(t)$ is negative definite provided that $\sigma_{2}^{2} / 2<\mu+\epsilon$. Therefore, we can have the following result (see [26]).

Corollary 3. Assume that $R_{0} \leq 1$ and $\sigma_{1}=0$. Then the disease-free equilibrium $E^{0}$ of system (2) is stochastically asymptotically stable in the large provided $\sigma_{2}^{2} / 2<\mu+\epsilon$.

\section{Existence of the Stationary Distribution}

It is well known that, in the study of epidemic dynamical model, the endemic equilibrium, which means that disease will prevail and persist in a population, is one of the most important and interesting topics owing to its theoretical and practical significance. Since $E^{*}$ is not an epidemic equilibrium of stochastic model (2), in this section, we turn to prove the existence of its stationary distribution. Before proving the main theorem of this section we first cite a known result from Hasmiskii [27] which will be useful to prove the theorem.
Let $X(t)$ be a regular time-homogeneous Markov process in $E_{l}$ (the $l$ dimensional Euclidean space) described by stochastic equation

$$
\mathrm{d} X(t)=b(X) \mathrm{d} t+\sum_{r=1}^{k} f_{r} \mathrm{~d} B_{r}(t)
$$

The diffusion matrix is defined as follows:

$$
A(x)=\left(a_{i j}(x)\right), \quad a_{i j}(x)=\sum_{r=1}^{k_{1}} f_{r}^{i}(x) f_{r}^{j}(x) .
$$

There exists a bounded domain $U \subset E_{l}$ with regular boundary $\Gamma$, having the following properties.

(B.1) In the domain $U$ and some neighborhood thereof, the smallest eigenvalue of the diffusion matrix $A(x)$ is bounded away from zero.

(B.2) If $x \in E_{l} \backslash U$, the mean time $\tau$ at which a path emerging from $x$ reaches the set $U$ is finite, and $\sup _{x \in K} E_{x} \tau<\infty$ for every compact subset $K \subset E_{l}$.

Lemma 4. If above assumptions (B.1) and (B.2) hold, then the Markov process $X(t)$ has a stationary distribution $\mu(\cdot)$. Let $f(\cdot)$ be a function integrable with respect to the measure $\mu$. Then

$$
P_{x}\left\{\lim _{T \rightarrow \infty} \int_{0}^{T} f(X(t)) d t=\int_{E_{l}} f(x) \mu(\mathrm{d} x)\right\}
$$

for all $x \in E_{l}$.

Remark 5. We can find the proof of Lemma 4 in [27]. Hasmiskii [27] refers the existence of a stationary distribution with suitable density function.

To validate (B.1), it suffices to prove $F$ is uniformly elliptical in $U$, where $F u=b(x) \cdot u_{x}+\left[\operatorname{tr} A(x) u_{x x}\right] / 2$; that is, there is a positive number $M$ such that

$$
\sum_{i, j=1}^{k} a_{i, j} \xi_{i} \xi_{j} \geq M|\xi|^{2}, \quad x \in U, \xi \in R^{k}
$$

(see [28] and Rayleigh's principle in [29]). To verify (B.2), it is sufficient to show that there exist some neighborhood $U$ and a nonnegative $C^{2}$-function such that and for any $E_{l} \backslash U, L V$ is negative function [30].

Theorem 6. Consider stochastic model (2) with initial condition in $(S(0), I(0)) \in \mathbb{R}_{+}^{2}$. Assume that $R_{0}>1$ and $0<\delta<\min \left(m_{1} S^{* 2}, m_{2} I^{* 2}\right)$ then there exists a stationary distribution $\mu(\cdot)$ for model (2). Here $\left(S^{*}, I^{*}\right)$ is the unique endemic equilibrium of (1) and

$$
\begin{gathered}
m_{1}=\mu-\sigma_{1}^{2}-\frac{\sigma_{3}^{2}(2 \mu+\epsilon) I^{*}}{\lambda}, \quad m_{2}=\mu+\epsilon-\sigma_{2}^{2}, \\
\delta=\sigma_{1}^{2} S^{* 2}+\left(I^{* 2}+\frac{2 \mu+\epsilon}{2 \lambda} I^{*}\right) \sigma_{2}^{2}+\frac{\sigma_{3}^{2} I^{*}(2 \mu+\epsilon)}{\lambda} S^{* 2} .
\end{gathered}
$$


Especially, we have

$$
\limsup _{t \rightarrow \infty} \frac{1}{t} \int_{0}^{t}\left[m_{1}\left(S(u)-S^{*}\right)^{2}+m_{2}\left(I(u)-I^{*}\right)^{2}\right] \mathrm{d} u \leq \delta .
$$

Proof. Our approach is inspired by the work of Ji and Jiang [31]. Since $R_{0}>1$, then there exists a unique positive equilibrium point $E^{*}=\left(S^{*}, I^{*}\right)$ of model (1). Furthermore, we have that

$$
\Lambda+\gamma I^{*}=\lambda S^{*} I^{*}+\mu S^{*}, \lambda S^{*} I^{*}=(\gamma+\mu+\epsilon) I^{*} .
$$

Define a positive definite function $V: E_{2} \rightarrow \mathbb{R}_{+}$:

$$
\begin{aligned}
V(t)= & \frac{1}{2}\left(S(t)+I(t)-S^{*}-I^{*}\right)^{2} \\
& +\frac{2 \mu+\epsilon}{\lambda}\left(I(t)-I^{*}-I^{*} \ln \frac{I(t)}{I^{*}}\right) \\
= & V_{1}(t)+\frac{2 \mu+\epsilon}{\lambda} V_{2}(t),
\end{aligned}
$$

where $E_{2}=\operatorname{Int}\left(\mathbb{R}^{2}+\right)$. Applying Itô's formula and using (31), we can calculate

$$
\begin{aligned}
\mathrm{d} V_{1}(t)= & \left(S(t)+I(t)-S^{*}-I^{*}\right)(\mathrm{d} S(t)+\mathrm{d} I(t)) \\
& +\frac{1}{2}(\mathrm{~d} S(t)+\mathrm{d} I(t))^{2} \\
= & L V_{1}(t)+\left(S(t)+I(t)-S^{*}-I^{*}\right) \\
& \times\left(\sigma_{1} S(t) \mathrm{d} B_{1}(t)+\sigma_{2} I(t) \mathrm{d} B_{2}(t)\right), \\
\mathrm{d} V_{2}(t)= & \left(1-\frac{I^{*}}{I(t)}\right) \mathrm{d} I(t)+\frac{I^{*}}{2 I^{2}(t)}(\mathrm{d} I(t))^{2} \\
= & L V_{2}(t)+\left(1-\frac{I^{*}}{I(t)}\right) \\
& \times\left(\sigma_{2} I(t) \mathrm{d} B_{2}(t)+\sigma_{3} S(t) I(t) \mathrm{d} B_{3}(t)\right),
\end{aligned}
$$

where

$$
\begin{aligned}
L V_{1}(t)= & \left(S(t)-S^{*}+I(t)-I^{*}\right)[\Lambda-\mu S(t)-(\mu+\epsilon) I(t)] \\
& +\frac{1}{2}\left(\sigma_{1}^{2} S^{2}(t)+\sigma_{2}^{2} I^{2}(t)\right) \\
= & \left(S(t)-S^{*}+I(t)-I^{*}\right) \\
& \times\left[\mu S^{*}+(\mu+\epsilon) I^{*}-\mu S(t)-(\mu+\epsilon) I(t)\right] \\
& +\frac{1}{2} \sigma_{1}^{2}\left(S(t)-S^{*}+S^{*}\right)^{2}+\frac{1}{2} \sigma_{2}^{2}\left(I(t)-I^{*}+I^{*}\right)^{2}
\end{aligned}
$$

$$
\begin{aligned}
& \leq\left(S(t)-S^{*}+I(t)-I^{*}\right) \\
& \times\left[-\mu\left(S(t)-S^{*}\right)-(\mu+\epsilon)\left(I(t)-I^{*}\right)\right] \\
& +\sigma_{1}^{2}\left(S(t)-S^{*}\right)^{2}+\sigma_{1}^{2} S^{* 2}+\sigma_{2}^{2}\left(I(t)-I^{*}\right)^{2}+\sigma_{2}^{2} I^{* 2} \\
& =-\left(\mu-\sigma_{1}^{2}\right)\left(S(t)-S^{*}\right)^{2} \\
& -(2 \mu+\epsilon)\left(S(t)-S^{*}\right)\left(I(t)-I^{*}\right) \\
& -\left(\mu+\epsilon-\sigma_{2}^{2}\right)\left(I(t)-I^{*}\right)^{2}+\sigma_{1}^{2} S^{* 2}+\sigma_{2}^{2} I^{* 2}, \\
& L V_{2}(t)=\left(I(t)-I^{*}\right)[\lambda S(t)-(\epsilon+\gamma+\mu)] \\
& +\frac{I^{*}}{2} \sigma_{2}^{2}+\frac{\sigma_{3}^{2} I^{*}}{2} S^{2}(t) \\
& =\left(I(t)-I^{*}\right)\left(\lambda S(t)-\lambda S^{*}\right) \\
& +\frac{I^{*}}{2} \sigma_{2}^{2}+\frac{\sigma_{3}^{2} I^{*}}{2}\left(S(t)-S^{*}+S^{*}\right)^{2} \\
& \leq \lambda\left(S(t)-S^{*}\right)\left(I(t)-I^{*}\right) \\
& +\frac{I^{*}}{2} \sigma_{2}^{2}+\sigma_{3}^{2} I^{*}\left(S(t)-S^{*}\right)^{2}+\sigma_{3}^{2} I^{*} S^{* 2} .
\end{aligned}
$$

It follows from (32)-(33) that

$$
\begin{aligned}
L V(t)= & L V_{1}(t)+\frac{2 \mu+\epsilon}{\lambda} L V_{2}(t) \\
\leq & -\left(\mu-\sigma_{1}^{2}\right)\left(S(t)-S^{*}\right)^{2}-\left(\mu+\epsilon-\sigma_{2}^{2}\right)\left(I(t)-I^{*}\right)^{2} \\
& +\sigma_{1}^{2} S^{* 2}+\sigma_{2}^{2} I^{* 2} \\
& +\frac{I^{*}(2 \mu+\epsilon)}{2 \lambda} \sigma_{2}^{2}+\frac{2 \mu+\epsilon}{\lambda} \sigma_{3}^{2} I^{*}\left(S-S^{*}\right)^{2} \\
& +\frac{2 \mu+\epsilon}{\lambda} \sigma_{3}^{2} I^{*} S^{* 2} \\
= & \left(\mu-\sigma_{1}^{2}-\frac{2 \mu+\epsilon}{\lambda} \sigma_{3}^{2} I^{*}\right)\left(S(t)-S^{*}\right)^{2} \\
& -\left(\mu+\epsilon-\sigma_{2}^{2}\right)\left(I(t)-I^{*}\right)^{2}+\sigma_{1}^{2} S^{* 2}+\sigma_{2}^{2} I^{* 2} \\
& +\frac{I^{*}(2 \mu+\epsilon)}{2 \lambda} \sigma_{2}^{2}+\frac{2 \mu+\epsilon}{\lambda} \sigma_{3}^{2} I^{*} S^{* 2} \\
\triangleq & -m_{1}\left(S(t)-S^{*}\right)^{2}-m_{2}\left(I(t)-I^{*}\right)^{2}+\delta
\end{aligned}
$$

where $m_{1}, m_{2}$, and $\delta$ are defined, respectively, in (29).

Notice that if $0<\delta<\min \left(m_{1} S^{* 2}, m_{2} I^{* 2}\right)$, then the ellipsoid

$$
-m_{1}\left(S(t)-S^{*}\right)^{2}-m_{2}\left(I(t)-I^{*}\right)^{2}+\delta=0
$$


lies entirely in $\mathbb{R}_{+}^{2}$. We can take $U$ to be any neighborhood of the ellipsoid such that $\bar{U} \subseteq E_{l}=\mathbb{R}_{+}^{2}$, so for $x \in U \backslash E_{l}, L V(t) \leq$ $-C$ ( $C$ is a positive constant), which implies condition (B.2) is satisfied.

On the other hand, we can write system (2) as the form of system (25):

$$
\begin{aligned}
\mathrm{d}\left(\begin{array}{c}
S(t) \\
I(t)
\end{array}\right)= & \left(\begin{array}{c}
\Lambda-\lambda S I-\mu S+\gamma I \\
\lambda S I-(\mu+\epsilon+\gamma) I
\end{array}\right) \mathrm{d} t+\left(\begin{array}{c}
\sigma_{1} S \\
0
\end{array}\right) \mathrm{d} B_{1} \\
& +\left(\begin{array}{c}
0 \\
\sigma_{2} I
\end{array}\right) \mathrm{d} B_{2}+\left(\begin{array}{c}
-\sigma_{3} S I \\
\sigma_{3} S I
\end{array}\right) \mathrm{d} B_{3}
\end{aligned}
$$

Here the diffusion matrix is

$$
A=\left(\begin{array}{cc}
\sigma_{1}^{2} S^{* 2}+\sigma_{3}^{2} S^{* 2} I^{* 2} & -\sigma_{3}^{2} S^{* 2} I^{* 2} \\
-\sigma_{3}^{2} S^{* 2} I^{* 2} & \sigma_{2}^{2} I^{* 2}+\sigma_{3}^{2} S^{* 2} I^{* 2}
\end{array}\right)
$$

There is an $M=\min \left\{\sigma_{1}^{2} S^{2}, \sigma_{2}^{2} I^{2}\right\}>0$, such that for all $\left(x_{1}, x_{2}\right) \in \bar{U}$ and $\xi \in \mathbb{R}^{2}$,

$$
\begin{aligned}
\sum_{i, j=1}^{2} a_{i j} \xi_{i} \xi_{j}= & \left(\sigma_{1}^{2} S^{2}+\sigma_{3}^{2} S^{2} I^{2}\right) \xi_{1}^{2} \\
& +\left(\sigma_{2}^{2} I^{2}+\sigma_{3}^{2} S^{2} I^{2}\right) \xi_{2}^{2}-2 \sigma_{3}^{2} S^{2} I^{2} \xi_{1} \xi_{2} \\
= & \sigma_{1}^{2} S^{2} \xi_{1}^{2}+\sigma_{2}^{2} I^{2} \xi_{2}^{2}+\sigma_{3}^{2} S^{2} I^{2}\left(\xi_{1}-\xi_{2}\right)^{2} \\
\geq & \sigma_{1}^{2} S^{2} \xi_{1}^{2}+\sigma_{2}^{2} I^{2} \xi_{2}^{2} \\
\geq & \min \left\{\sigma_{1}^{2} S^{2}, \sigma_{2}^{2} I^{2}\right\}|\xi|^{2} \\
= & M|\xi|^{2}
\end{aligned}
$$

which shows that condition (B.1) is also satisfied. Therefore, we can conclude that stochastic model (2) has a stationary distribution $\mu(\cdot)$.

Based on (30) in Theorem 6, we can further have the following persistence result of model (2).

Corollary 7. If $R_{0}>1$ and $\delta<\min \left\{m_{1}\left(S^{*}\right)^{2}, m_{2}\left(I^{*}\right)^{2}\right\}$, then model (2) is persistent in the mean.

Proof. Obviously, (30) holds. It follows that

$$
\begin{gathered}
\limsup _{t \rightarrow \infty} \frac{1}{t} \int_{0}^{t}\left(S(u)-S^{*}\right)^{2} \mathrm{~d} u \leq \frac{\delta}{m_{1}} \text { a.s., } \\
\limsup _{t \rightarrow \infty} \frac{1}{t} \int_{0}^{t}\left(I(u)-I^{*}\right)^{2} \mathrm{~d} u \leq \frac{\delta}{m_{2}} \text { a.s. }
\end{gathered}
$$

Noting also that

$$
2\left(S^{*}\right)^{2}-2 S^{*} S=2 S^{*}\left(S^{*}-S\right) \leq\left(S^{*}\right)^{2}+\left(S-S^{*}\right)^{2},
$$

we have that

$$
S \geq \frac{S^{*}}{2}-\frac{\left(S-S^{*}\right)^{2}}{2 S^{*}}
$$

It follows from (40) and (43) that

$$
\begin{aligned}
\liminf _{t \rightarrow \infty} \frac{1}{t} \int_{0}^{t} S(u) \mathrm{d} u & \geq \frac{S^{*}}{2}-\limsup _{t \rightarrow \infty} \int_{0}^{t} \frac{\left(S(u)-S^{*}\right)^{2}}{2 S^{*}} \mathrm{~d} u \\
& \geq \frac{S^{*}}{2}-\frac{\delta}{2 S^{*} m_{1}} \text { a.s. }
\end{aligned}
$$

Similarly, we have

$$
\begin{aligned}
\liminf _{t \rightarrow \infty} \frac{1}{t} \int_{0}^{t} I(u) \mathrm{d} u & \geq \frac{I^{*}}{2}-\limsup _{t \rightarrow \infty} \int_{0}^{t} \frac{\left(I(u)-I^{*}\right)^{2}}{2 I^{*}} \mathrm{~d} u \\
& \geq \frac{I^{*}}{2}-\frac{\delta}{2 I^{*} m_{2}} \text { a.s. }
\end{aligned}
$$

Therefore, model (2) is persistent in the mean.

\section{Extinction of the Disease}

In this section, our goal is to find the conditions under which the disease will go to extinction. In the previous section we have showed that under certain conditions, deterministic model (1) and the associated stochastic model (2) behave similarly in the sense that both have positive solutions which will not explode to infinity in a finite time. In other words, we show that under certain condition the noise will not spoil these properties. For the deterministic epidemic model (1), the value of the basic reproduction number $R_{0}$ determines the extinction or persistence of the disease: If $R_{0} \leq 1$, the disease will go to extinction, and if $R_{0}>1$, then the disease will be persistent in the population. However, we will show in this section that if the noise is sufficiently large, the disease will become extinct for stochastic model (2), although it may be persistent for its deterministic version (1). The following theorem gives a condition for the extinction of the disease expressed in terms of intensities of noise and system parameters.

Theorem 8. Consider stochastic model (2) with initial condition in $\mathbb{R}_{+}^{2}$. We have

$$
\limsup _{t \rightarrow \infty} \frac{\ln I(t)}{t} \leq \frac{\lambda^{2}}{2 \sigma_{3}^{2}}-(\gamma+\mu+\epsilon)-\frac{\sigma_{2}^{2}}{2} \text { a.s. }
$$

If $\lambda^{2} / 2 \sigma_{3}^{2} \leq(\gamma+\mu+\epsilon)+\sigma_{2}^{2} / 2$ holds, then $I(t)$ will go to zero exponentially with probability one. 

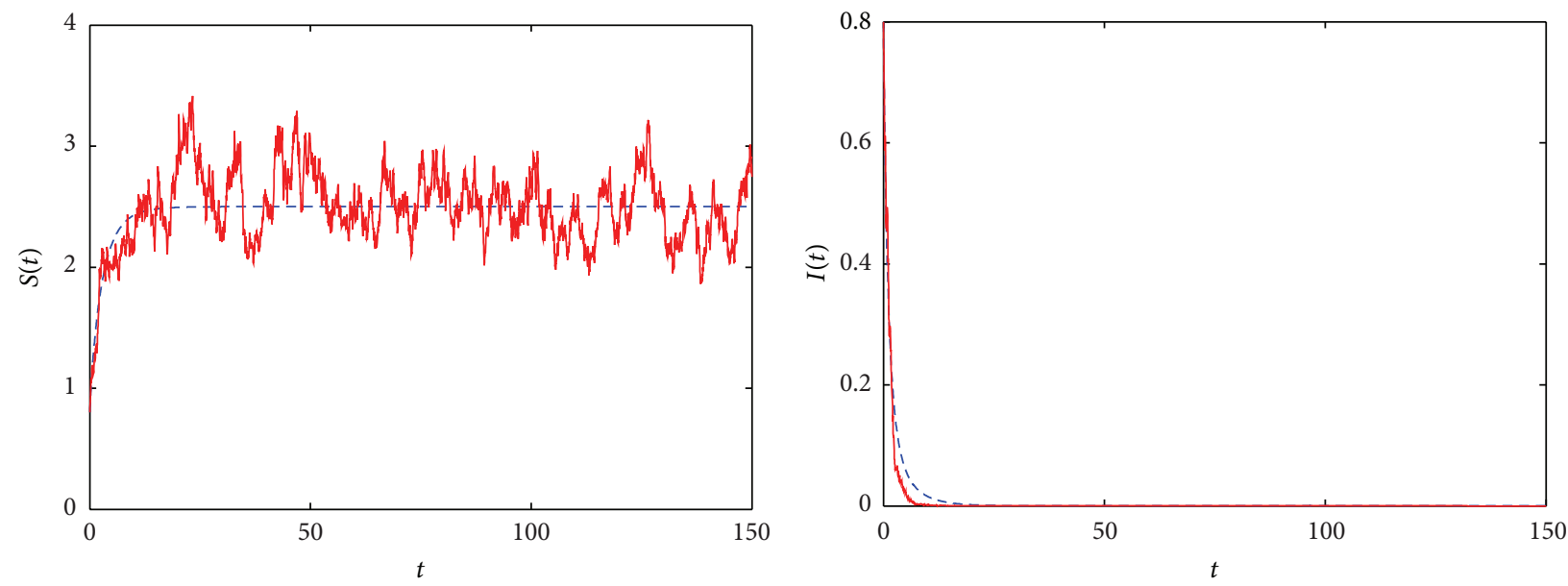

(a)
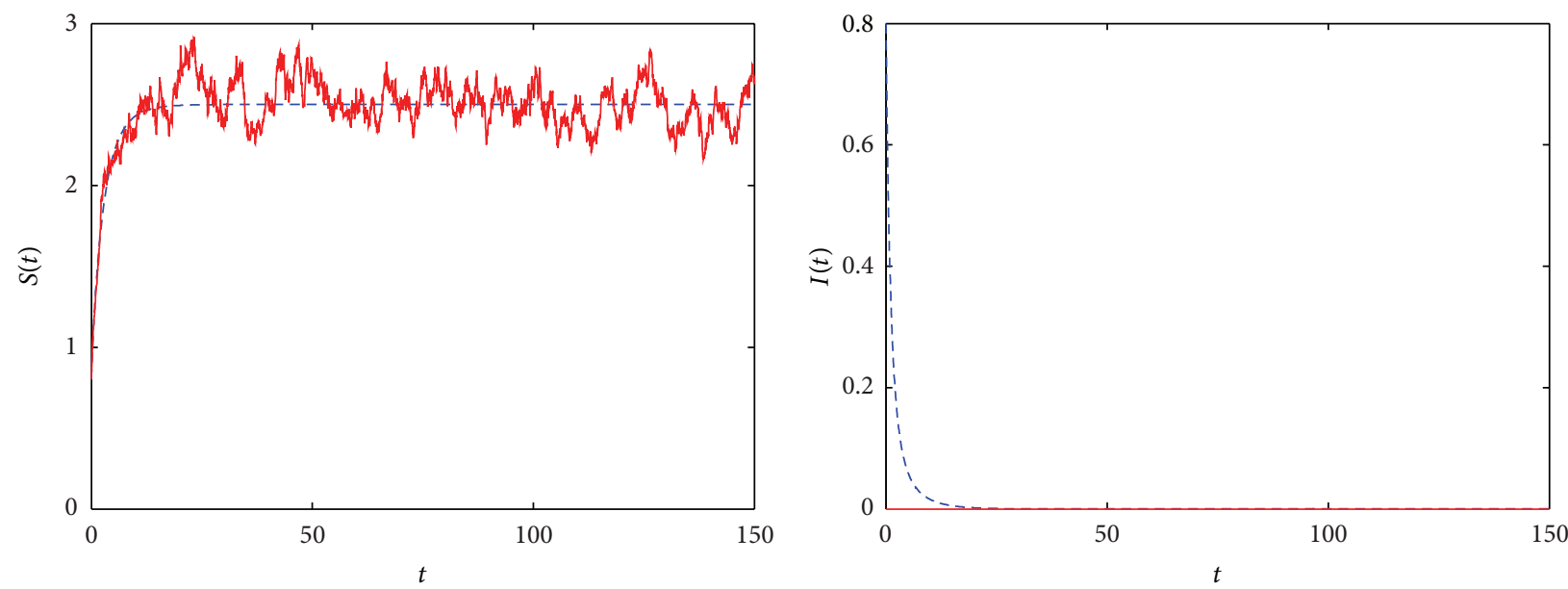

(b)

FIGURE 1: The trajectories of deterministic system (1) and stochastic system (2) with initial value $(S(0), I(0))=(0.8,0.8)$. Here $\Lambda=1, \lambda=$ $0.4, \mu=0.4, \epsilon=0.4$, and $\gamma=0.4$; (a) $\sigma_{1}=0.1, \sigma_{2}=0.1$, and $\sigma_{3}=0.1$; (b) $\sigma_{1}=0.05, \sigma_{2}=0.05$, and $\sigma_{3}=0.05$.

Proof. Define $V(I(t))=\ln I(t)$; by Itô's formula we have

$$
\begin{aligned}
\ln I(t)= & \ln I(0) \\
& +\int_{0}^{t}\left[\lambda S(u)-(\gamma+\mu+\epsilon)-\frac{\sigma_{2}^{2}}{2}-\frac{\sigma_{3}^{2} S^{2}(u)}{2}\right] \mathrm{d} u \\
& +\sigma_{2} B_{2}(t)+\int_{0}^{t} \sigma_{3} S(u) \mathrm{d} B_{3}(t) \\
= & \ln I(0) \\
& +\int_{0}^{t}\left[-\frac{\sigma_{3}^{2}}{2}\left(S^{2}(u)-\frac{2 \lambda}{\sigma_{3}^{2}} S(u)\right)\right. \\
& \left.\quad-(\gamma+\mu+\epsilon)-\frac{\sigma_{2}^{2}}{2}\right] \mathrm{d} u \\
& +\sigma_{2} B_{2}(t)+M(t) .
\end{aligned}
$$

Clearly, we have that

$$
-\frac{\sigma_{3}^{2}}{2}\left(S^{2}(t)-\frac{2 \lambda}{\sigma_{3}^{2}} S(t)\right)=-\frac{\sigma_{3}^{2}}{2}\left(S(t)-\frac{\lambda}{\sigma_{3}^{2}}\right)^{2}+\frac{\lambda^{2}}{2 \sigma_{3}^{2}} \leq \frac{\lambda^{2}}{2 \sigma_{3}^{2}} .
$$

It follows from (47) that

$$
\begin{aligned}
\ln I(t) \leq & \ln I(0)+\int_{0}^{t}\left[\frac{\lambda^{2}}{2 \sigma_{3}^{2}}-(\gamma+\mu+\epsilon)-\frac{\sigma_{2}^{2}}{2}\right] \mathrm{d} u \\
& +\sigma_{2} B_{2}(t)+M(t),
\end{aligned}
$$

where $M(t)=\int_{0}^{t} \sigma_{3} S(u) \mathrm{d} B_{3}(u)$ is a continuous local martingale whose quadratic variation is

$$
\langle M(t), M(t)\rangle=\sigma_{3}^{2} \int_{0}^{t} S^{2}(u) \mathrm{d} u,
$$

which implies that

$$
\limsup _{t \rightarrow \infty} \frac{\langle M, M\rangle_{t}}{t}<\infty .
$$



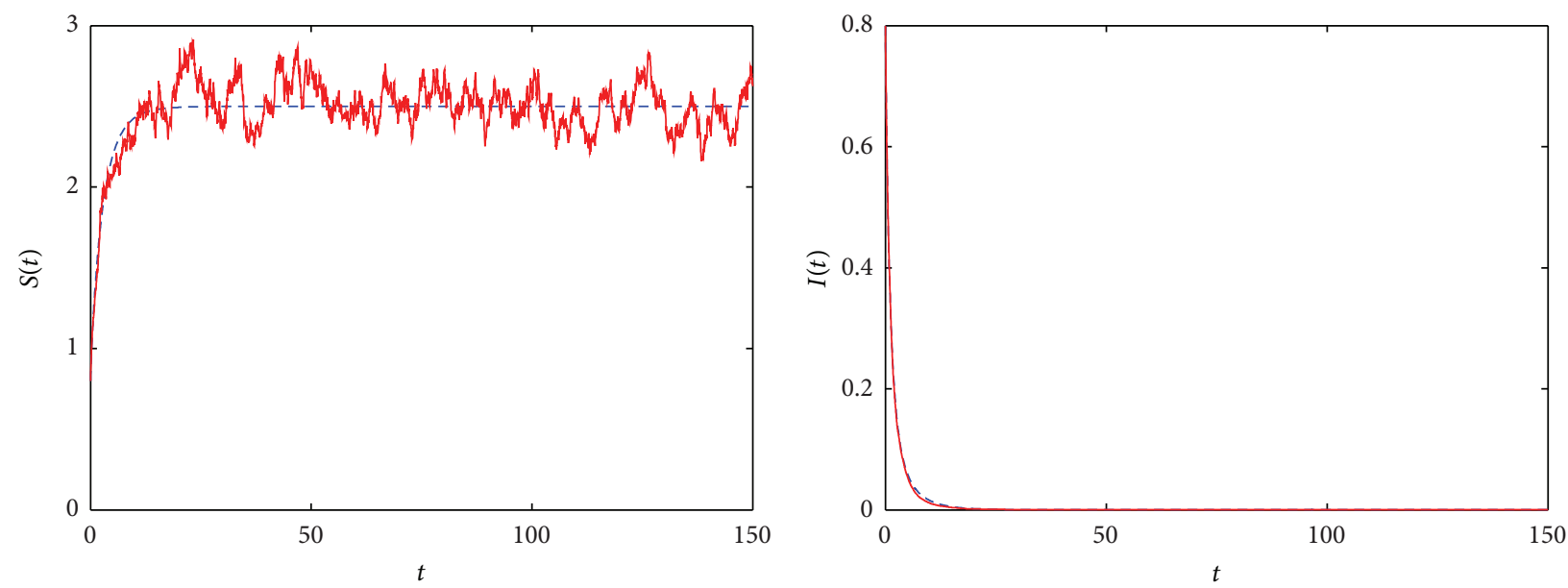

(a)
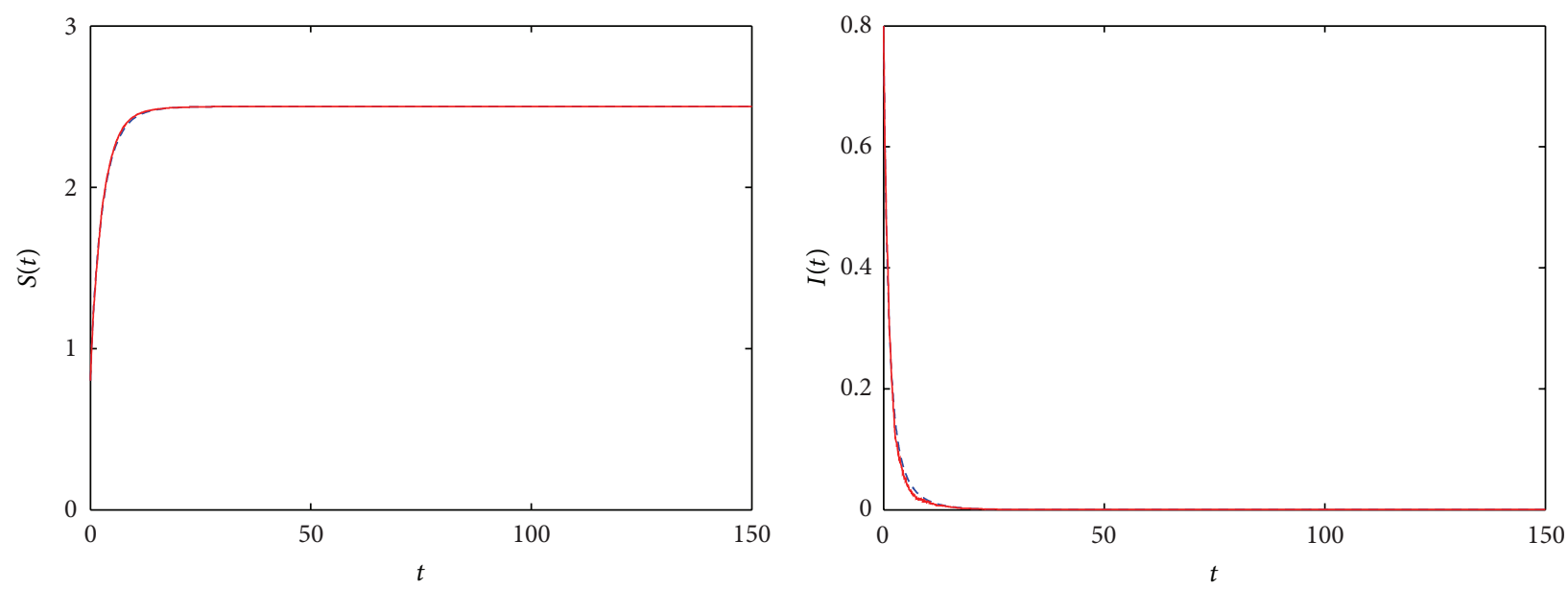

(b)

Figure 2: The trajectories of deterministic system (1) and stochastic system (2) with initial value $(S(0), I(0))=(0.8,0.8)$. Here $\Lambda=1, \lambda=0.4$, $\mu=0.4, \epsilon=0.4$, and $\gamma=0.4$; (a) $\sigma_{1}=0.05, \sigma_{2}=0.01$, and $\sigma_{3}=0.01$; (b) $\sigma_{1}=0, \sigma_{2}=0.01$, and $\sigma_{3}=0.01$.

Hence, by the strong law of large numbers of martingales,

$$
\lim _{t \rightarrow \infty} \frac{M(t)}{t}=0 \text { a.s. }
$$

Hence, we can divide both sides of (49) by $t$ and then let $t \rightarrow$ $\infty$ to obtain that

$$
\limsup _{t \rightarrow \infty} \frac{\ln I(t)}{t} \leq \frac{\lambda^{2}}{2 \sigma_{3}^{2}}-(\gamma+\mu+\epsilon)-\frac{\sigma_{2}^{2}}{2}
$$

The proof is therefore completed.

Remark 9. When $R_{0}>1$, the positive solution converges to the endemic equilibrium of deterministic model (1). However, the disease will die out exponentially regardless of the magnitude of $R_{0}$ provided that $\sigma_{2}$ and $\sigma_{3}$ are big enough such that $\lambda^{2} / 2 \sigma_{3}^{2} \leq(\gamma+\mu+\epsilon)+\sigma_{2}^{2} / 2$. That is to say, large noises can lead to the extinction of disease.

\section{Numerical Simulations}

In order to make the readers understand our results more better, we will perform some numerical simulations to illustrate our theoretical results. The numerical simulation is given by the following Milstein scheme [32]. Consider the discretization of model (2) for $t=0, \Delta t, 2 \Delta t, \ldots, n \Delta t$ :

$$
\begin{aligned}
S_{k+1}= & S_{k}+\left(\Lambda-\lambda S_{k} I_{k}-\mu S_{k}+\gamma I_{k}\right) \Delta t \\
& +S_{k}\left[\sigma_{1} \xi_{1, k} \sqrt{\Delta t}+\frac{1}{2} \sigma_{1}^{2}\left(\xi_{1, k}^{2}-1\right) \Delta t\right] \\
& +S_{k} I_{k}\left[\sigma_{3} \xi_{3, k} \sqrt{\Delta t}+\frac{1}{2} \sigma_{3}^{2}\left(\xi_{3, k}^{2}-1\right) \Delta t\right], \\
I_{k+1}= & I_{k}+\left[\lambda S_{k} I_{k}-(\gamma+\mu+\epsilon) I_{k}\right] \Delta t \\
& +I_{k}\left[\sigma_{2} \xi_{2, k} \sqrt{\Delta t}+\frac{1}{2} \sigma_{2}^{2}\left(\xi_{21, k}^{2}-1\right)\right] \Delta t \\
& +S_{k} I_{k}\left[\sigma_{3} \xi_{3, k} \sqrt{\Delta t}+\frac{1}{2} \sigma_{3}^{2}\left(\xi_{3, k}^{2}-1\right) \Delta t\right],
\end{aligned}
$$



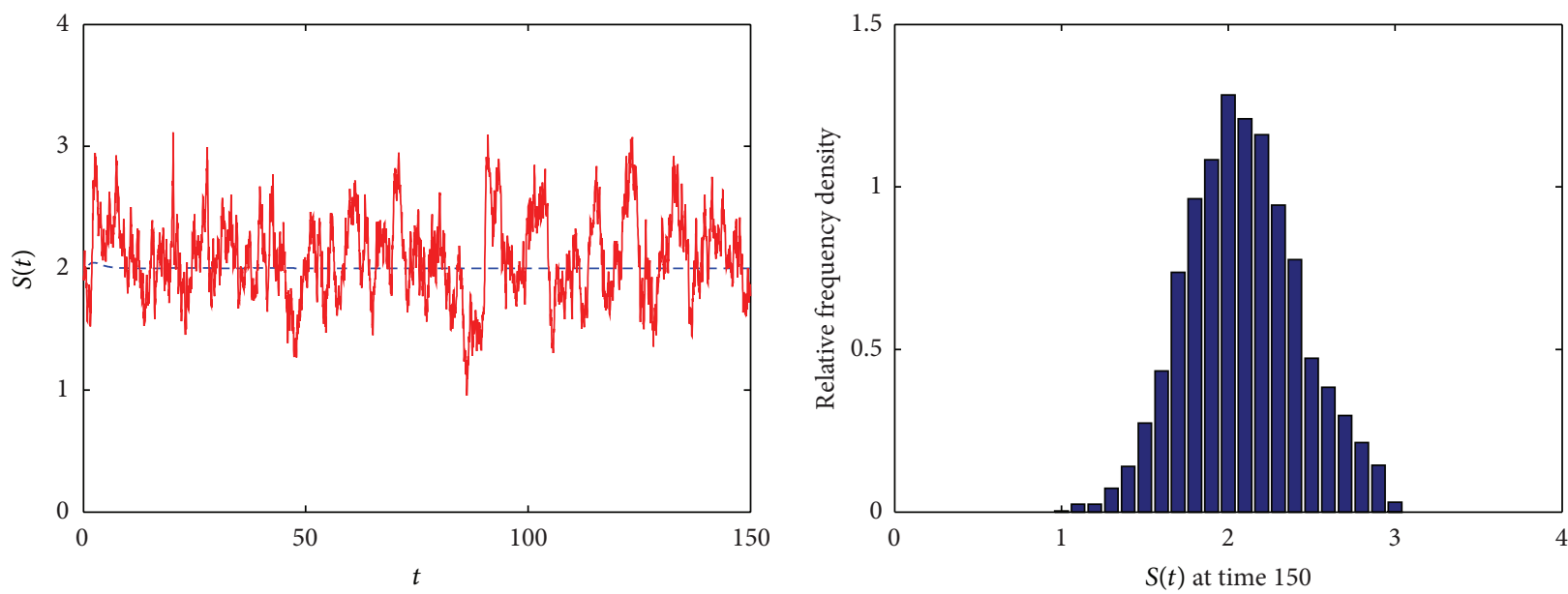

(a)
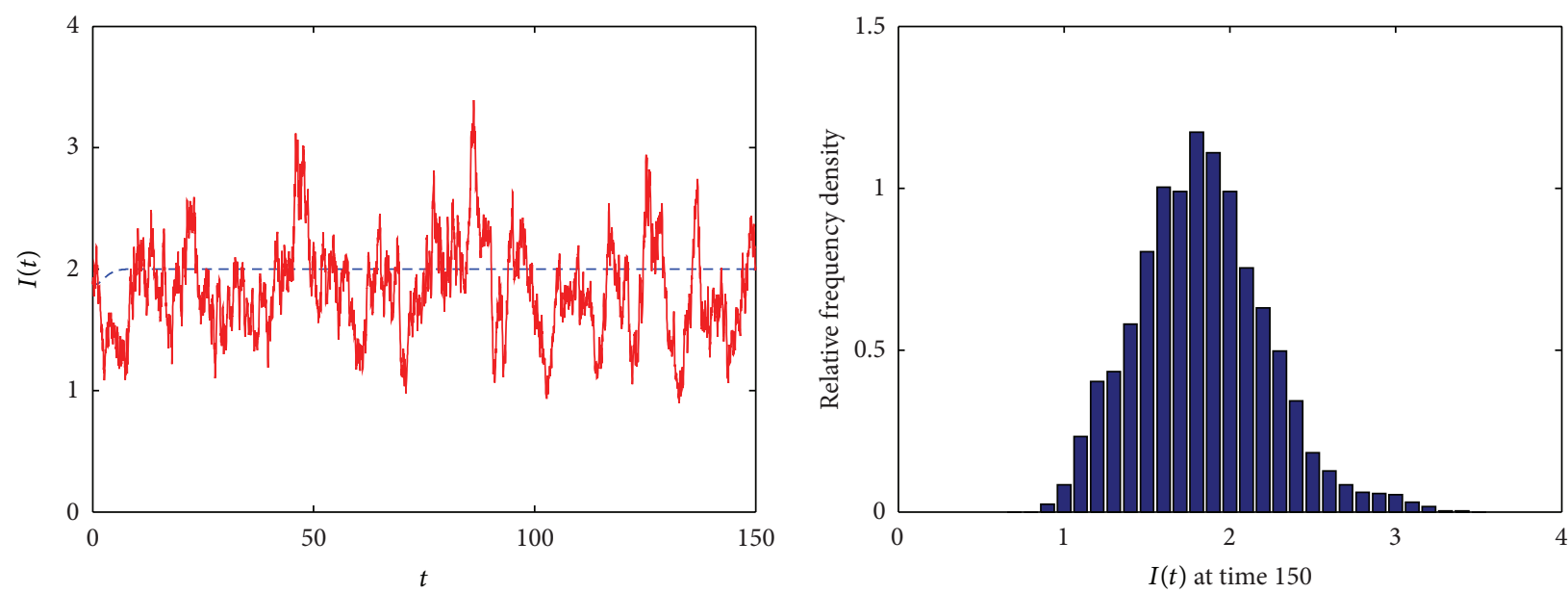

(b)

FIgURE 3: The solution of the stochastic system (2) and its histogram with initial value $(S(0), I(0))=(1.9,1.9)$. Here $\Lambda=2, \lambda=0.4, \mu=0.4$, $\epsilon=0.2$, and $\gamma=0.2 ; \sigma_{1}=0.1, \sigma_{2}=0.1$, and $\sigma_{3}=0.1$.

where $\Delta t$ is time increment and $\xi_{1, k}, \xi_{2, k}$, and $\xi_{3, k}(k=$ $1,2, \ldots, n)$ are independent Gaussian random variables $N(0,1)$ which can be generated numerically by pseudorandom number generators. In order to understand their role on the dynamics, we use different values of $\sigma_{1}, \sigma_{2}$, and $\sigma_{3}$. In all the following figures, the blue lines and the red lines represent solutions of the deterministic system (1) and the stochastic system (2), respectively.

In Figures 1 and 2, we always choose initial value $S(0)=$ $0.8, I(0)=0.8$, and parameters $\Lambda=1, \lambda=0.4, \mu=0.4, \epsilon=$ 0.4 , and $\gamma=0.4$ with different intensities of white noise which satisfy the conditions in Theorem 2 . We can easily compute that $R_{0}=0.8333<1, E^{0}=(\Lambda / \mu, 0)=(2.5,0)$. By Matlab software, we simulate the solution of model (2) with different values of $\sigma_{i}, i=1,2,3$ and the solution of model (1).

In Figure 1(a), we choose $\sigma_{1}=0.1, \sigma_{2}=0.1$, and $\sigma_{3}=0.1$, and in Figure 1(b), we choose $\sigma_{1}=0.05, \sigma_{1}=0.05$, and $\sigma_{3}=$ 0.05 . We can see from Figure 1 that the solution of model (2) will oscillate around the disease-free equilibrium in time, and moreover, the larger the intensities of the white noises are, the larger the fluctuations of the solutions will be.

Furthermore, if we take $\sigma_{1}$ the same value as in Figure 1(b), but take $\sigma_{2}, \sigma_{3}$ smaller values $\sigma_{2}=\sigma_{3}=0.01$. We can see from Figure 2(a) that the fluctuation of the solution will be very smaller. That is to say, the intensities of $\sigma_{2}$ and $\sigma_{3}$ have little effects on the solution.

When $\sigma_{1}=0$, then $E^{0}$ becomes the disease-free equilibrium of model (2). We can see from (20) that

$$
L V(t) \leq-0.4(S(t)-2.5)^{2}-0.79995 I^{2}(t) \leq 0 ;
$$

that is, the solution of model (2) is stochastically asymptotically stable in the large.

In the following, we consider the the long behavior of model (2) in the case of $R_{0}>1$. Choose the parameters

$$
\Lambda=2, \quad \lambda=0.4, \quad \mu=0.4, \quad \epsilon=0.2, \quad \gamma=0.2 \text {. }
$$



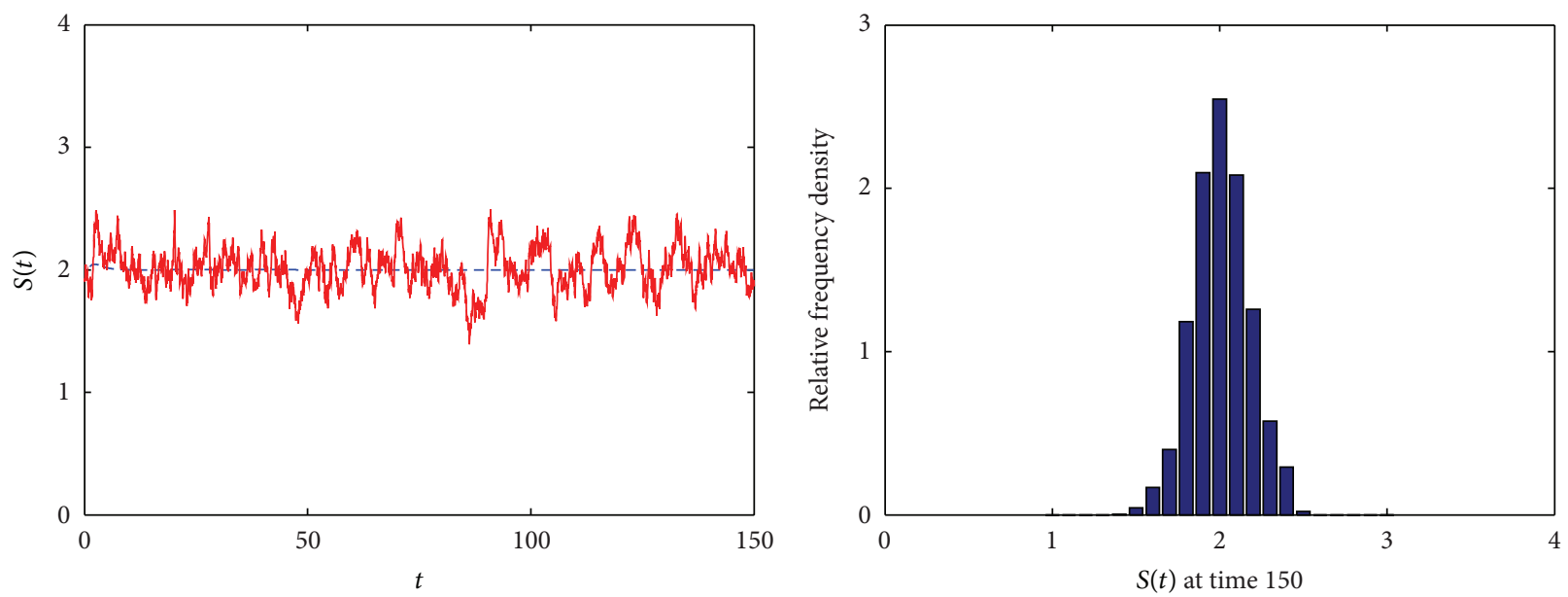

(a)
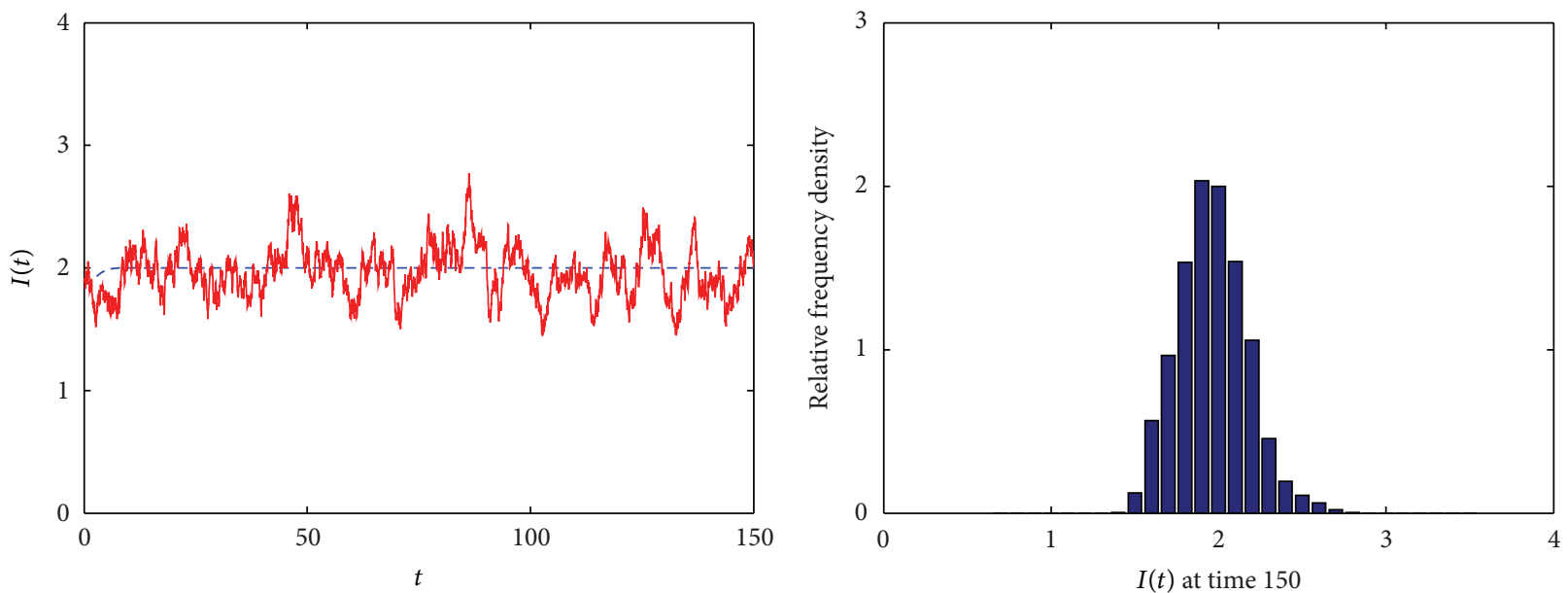

(b)

Figure 4: The solution of the stochastic system (2) and its histogram with initial value $(S(0), I(0))=(1.9,1.9)$. Here $\Lambda=2, \lambda=0.4, \mu=0.4$, $\epsilon=0.2$, and $\gamma=0.2 ; \sigma_{1}=0.05, \sigma_{2}=0.05$, and $\sigma_{3}=0.05$.

We can compute that $R_{0}=2.5, E^{*}=\left(S^{*}, I^{*}\right)=(2,2)$. We start our numerical simulation with environmental forcing strengths $\sigma_{1}=0.1, \sigma_{2}=0.1$, and $\sigma_{3}=0.1$ and starting from the initial point $(1.9,1.9)$. Figure 3 reports the result of one simulation run and stationary distribution about the populations of susceptibles and infectives. After some initial transients the population densities fluctuate around the deterministic steady-state values $S^{*}=2$ and $I^{*}=2$, respectively. It is to compute that $m_{1}=0.34, m_{2}=0.59$, $m_{1} S^{* 2}=1.46, m_{2} I^{* 2}=2.36$, and $\delta=0.305$. The conditions in Theorem 6 are satisfied. Accordingly, there exists a stationary distribution for model (2). From the histograms in Figure 3 we can see that the values of $S(t)$ and $I(t)$ are distributed normally around the mean values 2 and 2 , respectively.

Next we decrease intensities of environmental forcing to $\sigma_{1}=0.05, \sigma_{2}=0.05$, and $\sigma_{3}=0.05$ and again we observe that the population distribution fluctuates around the deterministic steady-state value but amplitude of fluctuation is less compared to earlier case (see Figure 4), which is also reflected in their stationary distributions. In Figure 4, the population of susceptible is distributed within $(1.5,2.6)$ and the population of infected remains within the range $(1.4,2.8)$, while in Figure 3 they are, respectively, distributed within (1, $3.1)$ and $(0.8,3.4)$.

Now, if we take $\sigma_{i}$ big enough, for example, $\sigma_{1}=0.1, \sigma_{2}=$ 0.1 , and $\sigma_{3}=0.33$, other parameters take the same values as in (56). We can verify that the conditions in Theorem 8 are satisfied. Moreover, we have

$$
\limsup _{t \rightarrow \infty} \frac{\ln I(t)}{t} \leq-0.0704,
$$

which means that $I(t)$ will go to zero exponentially with time (see Figure 5). That is to say, large noises can lead the disease to extinction, which is a phenomenon different from its corresponding deterministic model (1).

\section{Acknowledgments}

The authors would like to acknowledge the support from the National Natural Science Foundation of China (11271260 and 


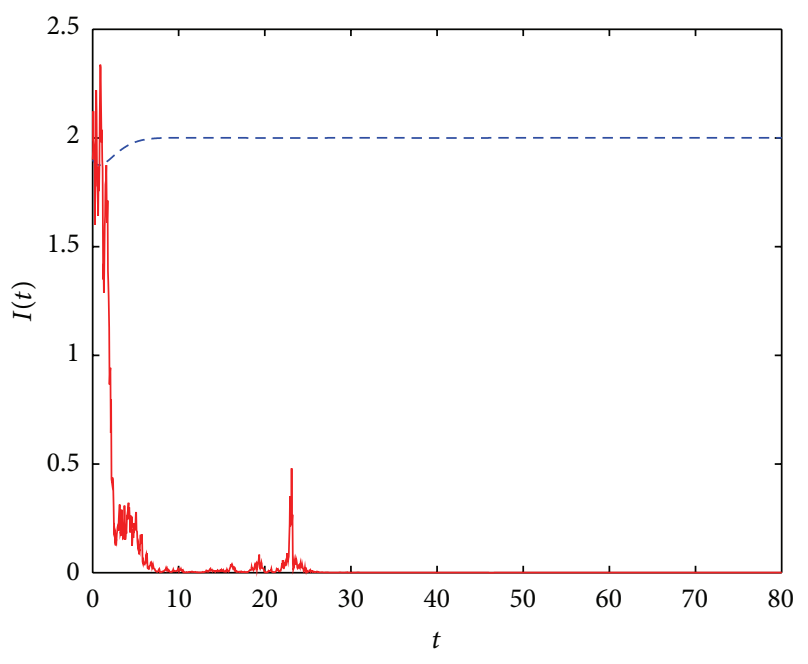

FIGURE 5: Computer simulation of a single path of $I(t)$ for the SDE model (2) with initial value $(S(0), I(0))=(1.9,1.9)$ and its corresponding deterministic model (1) for parameter values $\Lambda=2$, $\lambda=0.4, \mu=0.4, \epsilon=0.2, \gamma=0.2, \sigma_{1}=0.1, \sigma_{2}=0.1$, and $\sigma_{3}=0.33$.

11071164) and the Innovation Program of Shanghai Municipal Education Commission (13ZZ116).

\section{References}

[1] V. Capasso and G. Serio, "A generalization of the KermackMcKendrick deterministic epidemic model," Mathematical Biosciences, vol. 42, no. 1-2, pp. 43-61, 1978.

[2] W. M. Liu, S. A. Levin, and Y. Iwasa, "Influence of nonlinear incidence rates upon the behavior of SIRS epidemiological models," Journal of Mathematical Biology, vol. 23, no. 2, pp. 187204, 1986.

[3] H. W. Hethcote, "Qualitative analyses of communicable disease models," Mathematical Biosciences, vol. 28, no. 3-4, pp. 335-356, 1976.

[4] D. Xiao and S. Ruan, "Global analysis of an epidemic model with nonmonotone incidence rate," Mathematical Biosciences, vol. 208, no. 2, pp. 419-429, 2007.

[5] S. Ruan and W. Wang, "Dynamical behavior of an epidemic model with a nonlinear incidence rate," Journal of Differential Equations, vol. 188, no. 1, pp. 135-163, 2003.

[6] W. M. Liu, H. W. Hethcote, and S. A. Levin, "Dynamical behavior of epidemiological models with nonlinear incidence rates," Journal of Mathematical Biology, vol. 25, no. 4, pp. 359380, 1987.

[7] Y. Jin, W. Wang, and S. Xiao, "An SIRS model with a nonlinear incidence rate," Chaos, Solitons and Fractals, vol. 34, no. 5, pp. 1482-1497, 2007.

[8] Y. Yang and D. Xiao, "Influence of latent period and nonlinear incidence rate on the dynamics of SIRS epidemiological models," Discrete and Continuous Dynamical Systems B, vol. 13, no. 1, pp. 195-211, 2010.

[9] R. Xu and Z. Ma, "Global stability of a delayed SEIRS epidemic model with saturation incidence rate," Nonlinear Dynamics, vol. 61, no. 1-2, pp. 229-239, 2010.
[10] R. Xu and Z. Ma, "Global stability of a SIR epidemic model with nonlinear incidence rate and time delay," Nonlinear Analysis. Real World Applications, vol. 10, no. 5, pp. 3175-3189, 2009.

[11] H. W. Hethcote and P. van den Driessche, "Some epidemiological models with nonlinear incidence," Journal of Mathematical Biology, vol. 29, no. 3, pp. 271-287, 1991.

[12] G. Chen and T. Li, "Stability of stochastic delayed SIR model," Stochastics and Dynamics, vol. 9, no. 2, pp. 231-252, 2009.

[13] E. Tornatore, S. M. Buccellato, and P. Vetro, "Stability of a stochastic SIR system," Physica A, vol. 354, no. 1-4, pp. 111-126, 2005.

[14] C. Ji, D. Jiang, Q. Yang, and N. Shi, "Dynamics of a multigroup SIR epidemic model with stochastic perturbation," Automatica, vol. 48, no. 1, pp. 121-131, 2012.

[15] B. Frank and N. Peter, "A general model for stochastic SIR epidemics with two levels of mixing," Mathematical Biosciences, vol. 180, pp. 73-102, 2002.

[16] J. Yu, D. Jiang, and N. Shi, "Global stability of two-group SIR model with random perturbation," Journal of Mathematical Analysis and Applications, vol. 360, no. 1, pp. 235-244, 2009.

[17] I. Nåsell, "Stochastic models of some endemic infections," Mathematical Biosciences, vol. 179, no. 1, pp. 1-19, 2002.

[18] Q. Yang, D. Jiang, N. Shi, and C. Ji, "The ergodicity and extinction of stochastically perturbed SIR and SEIR epidemic models with saturated incidence," Journal of Mathematical Analysis and Applications, vol. 388, no. 1, pp. 248-271, 2012.

[19] M. Carletti, "Mean-square stability of a stochastic model for bacteriophage infection with time delays," Mathematical Biosciences, vol. 210, no. 2, pp. 395-414, 2007.

[20] A. Lahrouz and L. Omari, "Extinction and stationary distribution of a stochastic SIRS epidemic model with non-linear incidence," Statistics \& Probability Letters, vol. 83, no. 4, pp. 960968, 2013.

[21] W. O. Kermack and A. G. McKendrick, "Contributions to the mathematical theory of epidemics," Proceedings of the Royal Society A, vol. 115, pp. 700-721, 1927.

[22] D. Jiang, C. Ji, N. Shi, and J. Yu, "The long time behavior of DI SIR epidemic model with stochastic perturbation," Journal of Mathematical Analysis and Applications, vol. 372, no. 1, pp. 162-180, 2010.

[23] W. Y. Tan and X. Zhu, "A stochastic model of the HIV epidemic for heterosexual transmission involving married couples and prostitutes-I. The probabilities of HIV transmission and pair formation," Mathematical and Computer Modelling, vol. 24, no. 11, pp. 47-107, 1996.

[24] N. Dalal, D. Greenhalgh, and X. Mao, "A stochastic model of AIDS and condom use," Journal of Mathematical Analysis and Applications, vol. 325, no. 1, pp. 36-53, 2007.

[25] J. R. Beddington and R. M. May, "Harvesting natural populations in a randomly fluctuating environment," Science, vol. 197, no. 4302, pp. 463-465, 1977.

[26] X. Mao, Stochastic Differential Equations and Applications, Horwood, Chichester, UK, 1997.

[27] R. Z. Hasminskii, Stochastic Stability of Differential Equations, Sijthoff \& Noordhoff, 1980.

[28] T. C. Gard, Introduction to Stochastic Differential Equations, vol. 114, Marcel Dekker, New York, NY, USA, 1987.

[29] G. Strang, Linear Algebra and Its Applications, Thomson Learning, 1988. 
[30] C. Zhu and G. Yin, "Asymptotic properties of hybrid diffusion systems," SIAM Journal on Control and Optimization, vol. 46, no. 4, pp. 1155-1179, 2007.

[31] C. Ji and D. Jiang, "Dynamics of a stochastic density dependent predator-prey system with Beddington-DeAngelis functional response," Journal of Mathematical Analysis and Applications, vol. 381, no. 1, pp. 441-453, 2011.

[32] D. J. Higham, "An algorithmic introduction to numerical simulation of stochastic differential equations," SIAM Review, vol. 43, no. 3, pp. 525-546, 2001. 


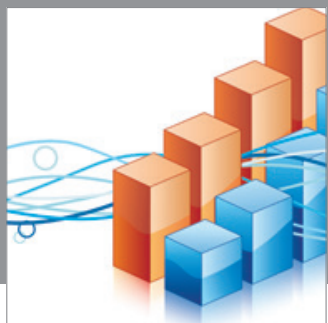

Advances in

Operations Research

mansans

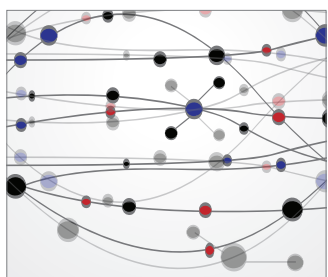

The Scientific World Journal
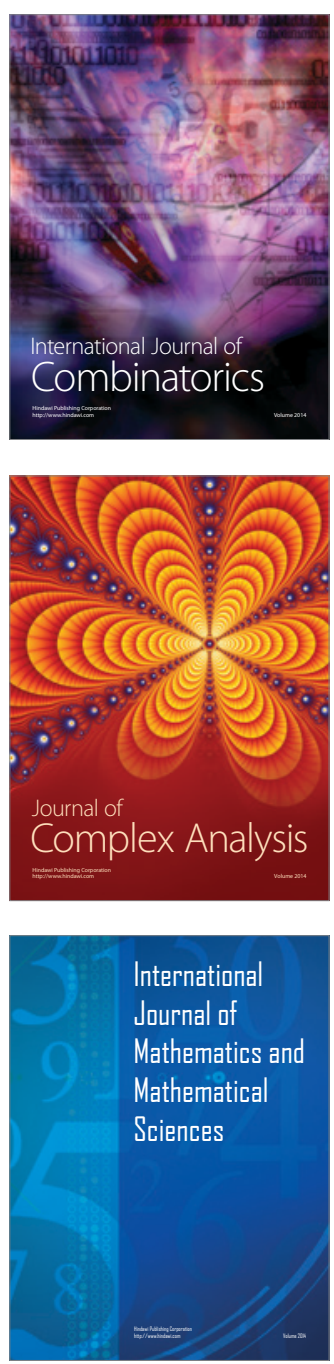
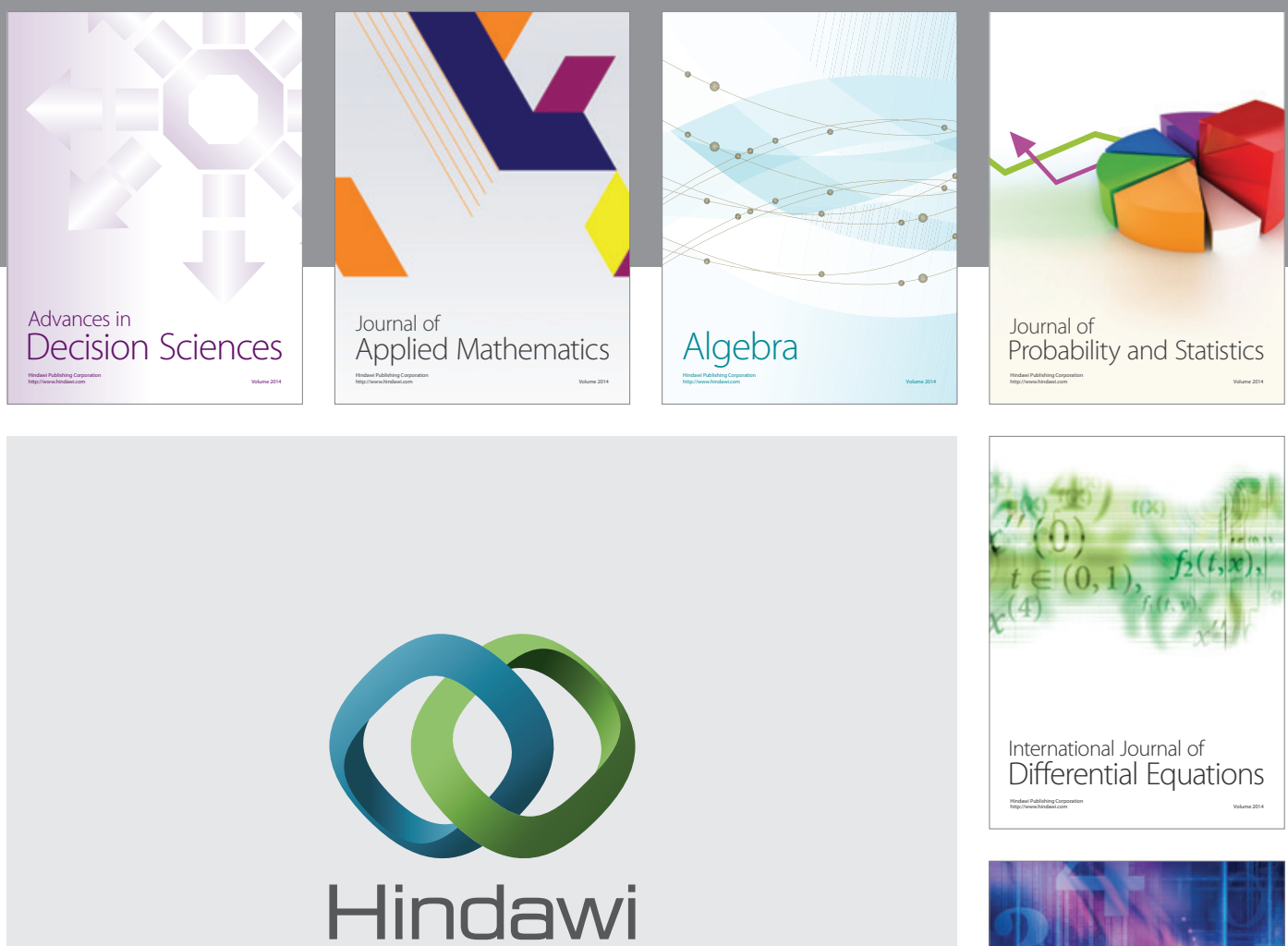

Submit your manuscripts at http://www.hindawi.com
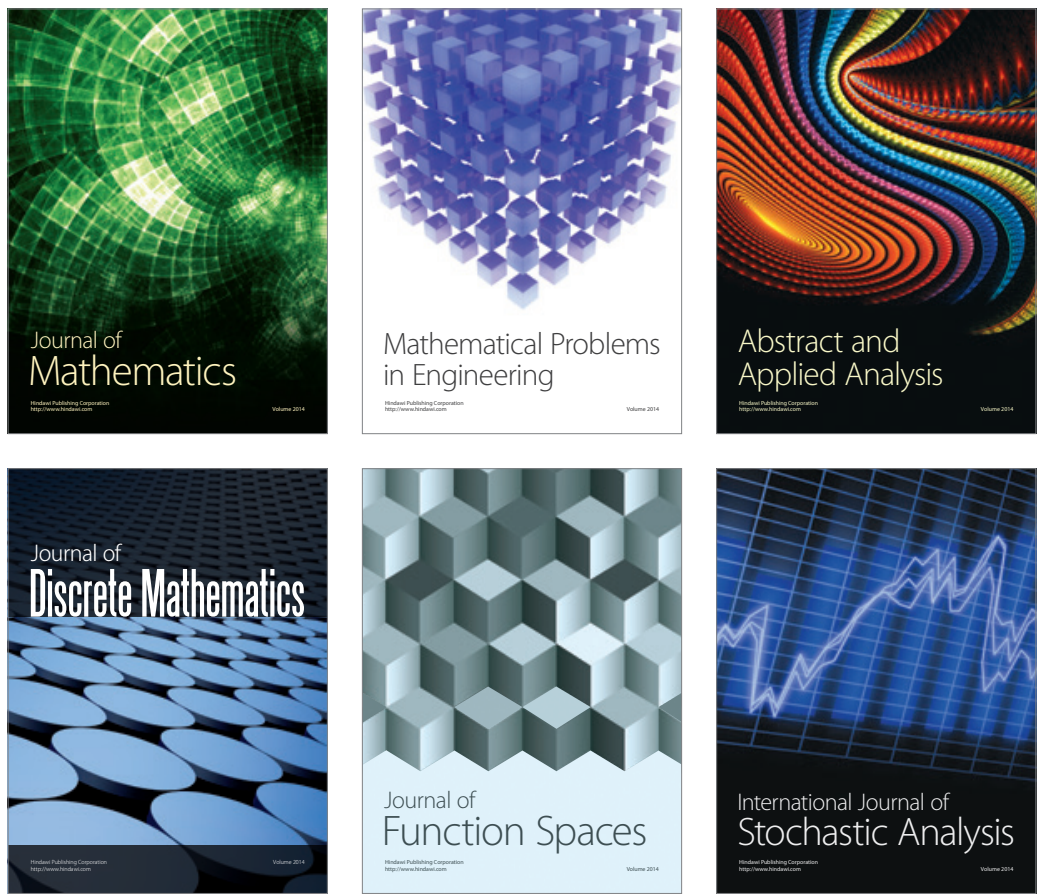

Journal of

Function Spaces

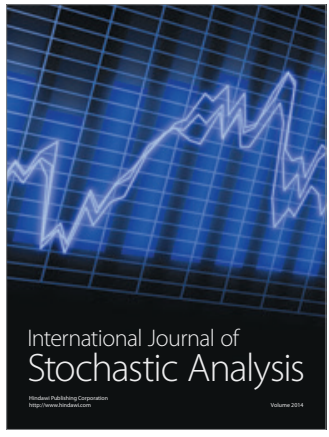

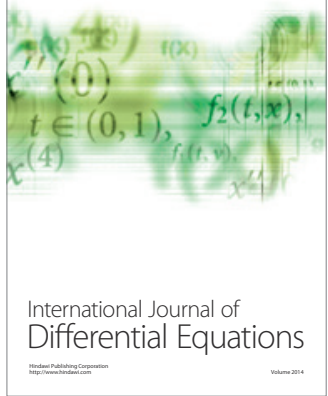
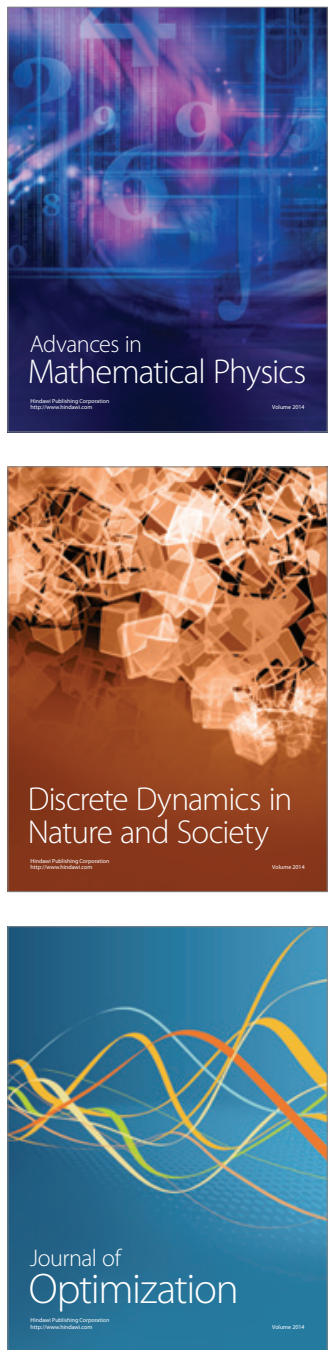